\title{
Local Extinction of Diffusion Flames in Fires
}

\author{
VIVIEN R. LECOUSTRE, PRAVEEN NARAYANAN, HOWARD R. BAUM, and ARNAUD \\ TROUVÉ \\ Department of Fire Protection Engineering \\ University of Maryland \\ College Park, MD 20742, USA
}

\begin{abstract}
The objective of the present study is to use large activation energy asymptotic (AEA) theory to bring basic information on the extinction limits of non-premixed flames. The AEA analysis leads to an explicit expression that predicts the occurrence of flame extinction in the form of a critical Damköhler number criterion; the criterion provides a unified framework to explain the different extinction limits that are observed in non-premixed combustion (i.e., aerodynamic quenching, thermal quenching, and dilution quenching). The critical Damköhler number criterion is then formulated in terms of six input variables; these variables characterize the magnitude of flame stretch, the magnitude of the flame heat losses, and the composition and heat content of the fuel and oxidizer supply streams; these input variables thereby contain information on (laminar or turbulent) flow-induced perturbations, deviations from adiabatic combustion, and air and fuel vitiation. Different two-dimensional flammability maps are then presented using different assumptions aimed at reducing the dimension of the parameter space from six to two. While providing a limited view point, these flammability maps provide valuable insights; it is found for instance that diffusion flames are more sensitive to air vitiation than fuel vitiation.
\end{abstract}

KEYWORDS: extinction, combustion, diffusion flame, flame stretch, vitiation, radiation, modeling.

\section{INTRODUCTION}

Total flame extinction is routinely observed in fire configurations, for instance as a result of fuel burn-out, oxygen depletion, or possibly following the activation of a fire suppression system. Total flame extinction is a system-level event that may be viewed as the result of the cumulative action of individual flame-level events known as local flame extinction. The exact weight of local flame extinction phenomena in fire dynamics depends on a number of factors, including the intensity of the fuel supply to the flame zone (i.e., the rate of production of flammable vapors by fuel sources), the quality of the fuel supply to the flame zone (i.e., the composition of the flammable vapors produced by fuel sources), the intensity of the oxygen supply to the flame zone (i.e., the air ventilation capacity of the flame zone), the sooting propensity of the fuel vapors (i.e., the rate of formation of soot particles in the flame zone), and the possible presence of fire suppressants (e.g., inert gaseous agents or liquid water sprays). In fire configurations characterized by good fuel supply, good ventilation and low soot loading conditions, the flame zone typically corresponds to a quasi-complete non-premixed combustion regime, i.e., to quasi-unity combustion efficiencies and low probabilities of occurrence of flame extinction. In contrast, in fire configurations characterized by poor fuel supply, poor ventilation or high soot loading conditions, or in configurations characterized by the presence of fire suppressants, the flame zone often corresponds to a more complex combustion regime featuring significant deviations from complete combustion and moderate-to-high probabilities of occurrence of local flame extinction. In such configurations, flame extinction is responsible for reduced levels of the heat release rate and increased levels of emission of products of incomplete combustion (e.g., carbon monoxide, unburnt hydrocarbons, and soot); the reduced levels of heat release rate correspond in turn to a decreased risk of fire spread; the increased levels of carbon monoxide and unburnt hydrocarbons emission correspond to an increased risk of toxic poisoning and in some cases, to an increased risk of explosive combustion (e.g., backdraft phenomena).

Flame extinction is a classical topic in combustion science [1-3]. Diffusion flames may be extinguished by a number of different mechanisms. For instance, diffusion flames may be extinguished by aerodynamic quenching, a mechanism in which the flame is weakened by fast flow-induced perturbations and a critical decrease in the flame residence time. Diffusion flames may also be extinguished by thermal quenching, a mechanism in which the flame is weakened by heat losses (e.g., radiant cooling, convective cooling to cold wall surfaces, or water evaporative cooling in fire suppression applications). Finally, diffusion flames may 
be extinguished by dilution quenching, a mechanism in which the flame is weakened due to changes in the composition of the fuel or oxidizer supply stream (e.g., air vitiation in under-ventilated fires). In both thermal and dilution quenching, extinction occurs because of a critical increase in the flame chemical time.

Laminar flame theory suggests that all these different phenomena may be explained by a single flame extinction criterion known as a Damköhler number criterion [1-3]: the Damköhler number $D a$ is defined as the ratio of a characteristic fuel-oxidizer mixing time divided by a characteristic chemical time, $D a=\left(\tau_{\text {mixing }} / \tau_{\text {chemical }}\right)$, and extinction is predicted to occur for values of $D a$ that are critically low. Theoretical analysis shows that the characteristic mixing time $\tau_{\text {mixing }}$ scales like the inverse of flame stretch, with flame stretch defined as the stoichiometric value of the scalar dissipation rate $\chi_{\text {st }}\left(\chi_{\text {st }}\right.$ gives a measure of the rate of fuel-oxidizer mixing at the flame location); theoretical analysis also shows that the characteristic chemical time $\tau_{\text {chemical }}$ scales like $\exp \left(T_{a} / T_{\text {st }}\right)$, where $T_{\text {st }}$ is the flame temperature and $T_{a}$ an activation temperature (assuming Arrhenius-like combustion chemistry, $T_{a}$ gives a measure of the sensitivity of the combustion chemistry to changes in temperature). Thus, we find that the Damköhler number is a function of flame stretch and flame temperature, $D a \sim\left(1 / \chi_{s t}\right) / \exp \left(T_{a} / T_{\text {st }}\right)$. Critically low values of $D a$ may therefore be achieved in several ways [4-13]: low values of $D a$ may be achieved under fast mixing conditions (i.e., when $\chi_{\text {st }}$ is large and $\tau_{\text {mixing }}$ is short); low values of $D a$ may also be achieved under low temperature conditions (i.e., when $T_{\text {st }}$ is low and $\tau_{\text {chemical }}$ is long). The fast mixing limit is the extinction limit associated with aerodynamic quenching; the low temperature limit is the extinction limit associated with thermal quenching and dilution quenching.

We examine in the present study the classical extinction theory of laminar diffusion flames based on the concept of a critical Damköhler number. This theory provides a unified framework to explain the different mechanisms associated with laminar flame extinction (aerodynamic, thermal, and dilution quenching); consistent with the flamelet perspective [1-3], it also provides a description of extinction in turbulent diffusion flames. The present study is a continuation of earlier work presented in Ref. [14] in which we perform a large activation energy asymptotic (AEA) analysis and study the extinction limits of laminar counter-flow ethylene-air diffusion flames as a function of flame stretch and radiant losses (with contributions from both gas and soot radiation). The specific objectives of the present study are to develop a parametrization of the AEA flame extinction criterion and to construct flammability maps. Another objective is to extend the earlier work presented in Ref. [14] to the case of vitiated fuel and air supply streams; in the following, we consider the extinction limits of flames supplied with different ethylenenitrogen and air-nitrogen mixtures and at different temperatures.

The AEA configuration is a classical counter-flow flame, herein referred to as a strained laminar flame (SLF); the AEA configuration is presented in the next section and is followed by a discussion of the response of SLF flames to changes in both flame stretch and composition of the fuel or oxidizer supply stream. The paper then turns to an analysis of the extinction criterion and extinction limits of SLF flames, including a discussion of the effects of flame stretch (aerodynamic quenching), radiant losses (thermal quenching), and nitrogen dilution (dilution quenching); the results are presented in the form of flammability maps. Conclusions are summarized at the end of the paper.

\section{STRAINED LAMINAR FLAMES (SLF)}

The SLF configuration corresponds to a classical, laminar, steady, one-dimensional, planar, counter-flow, diffusion flame (Fig. 1). The fuel is ethylene and the oxidizer is air. The AEA analysis presented in Ref. [14] uses a classical two-layer decomposition of the flame structure into an inner reaction zone and an outer mixing zone. The inner reaction zone is described using asymptotic expansions in terms of a small parameter related to an inverse Zeldovich number; the outer mixing zone is a convection-diffusion chemically-inert zone modified by thermal radiation. The AEA analysis assumes single-step global combustion chemistry, constant heat capacity, and unity Lewis numbers; it also includes a two-equation phenomenological model to describe soot formation, growth, and oxidation processes, as well as a generalized treatment of thermal radiation that considers radiation from both gaseous species $\left(\mathrm{CO}_{2}\right.$ and $\mathrm{H}_{2} \mathrm{O}$ ) and soot particles, assumes spectrally-averaged gray-medium properties, and applies to flames with an arbitrary optical thickness. The reader is referred to Ref. [14] for more details.

Figure 2 presents a typical SLF flame structure, as predicted by the AEA analysis. Figure 2a presents the spatial variations of mixture fraction in a direction normal to the flame. Mixture fraction is equal to 1 on the 


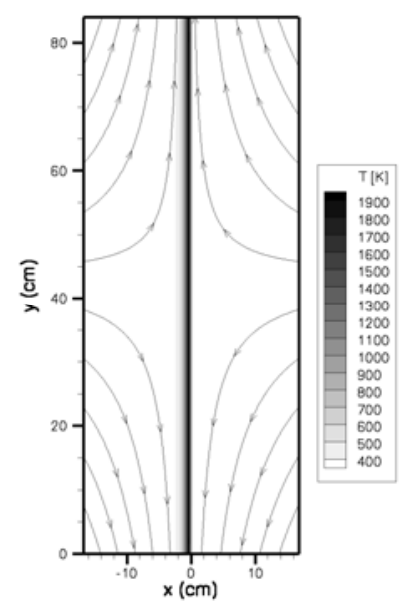

Fig. 1. Laminar counter-flow diffusion flame. The fuel (oxidizer) supply stream is flowing from the left (right); the flame is the thin region located near the stagnation plane at $x=0$. The plot shows selected flow streamlines and temperature iso-contours. Case, $\alpha=6.5 \mathrm{~s}^{-1}\left(\chi_{\mathrm{st}}=0.2 \mathrm{~s}^{-1}\right)$.

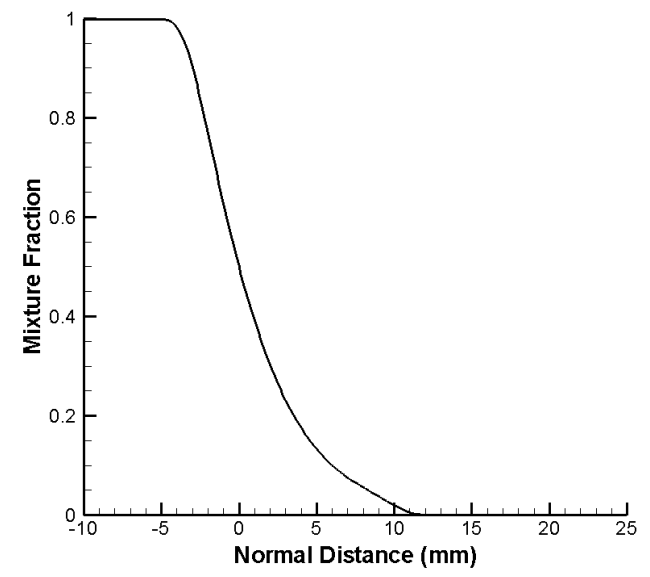

(a)

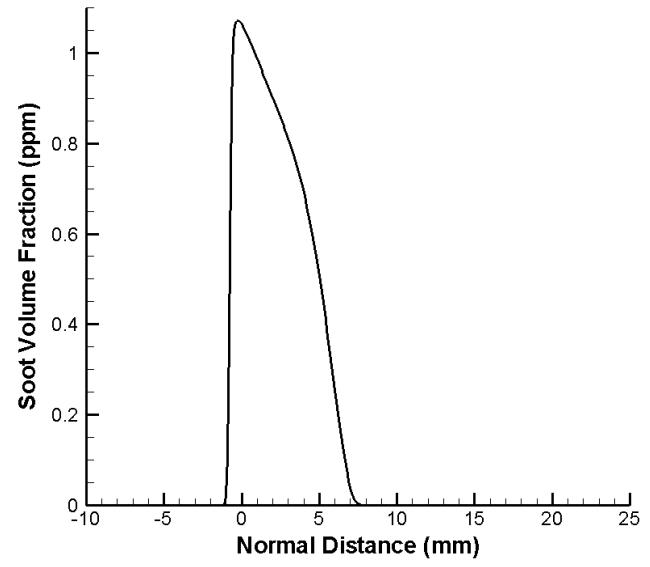

(c)

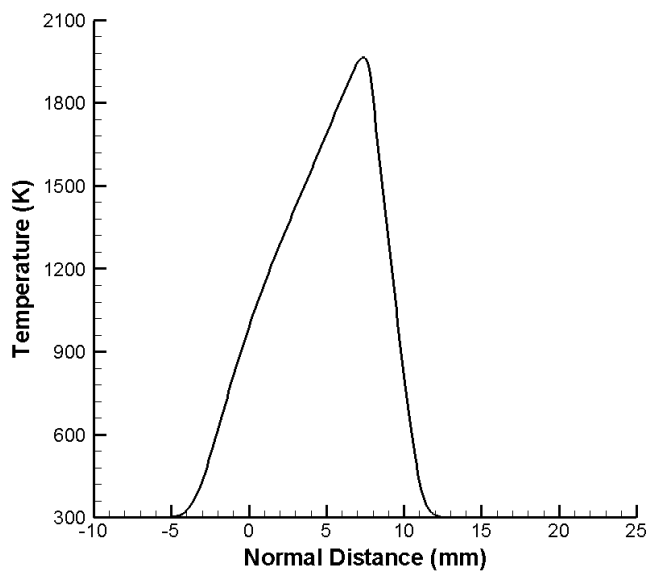

(b)

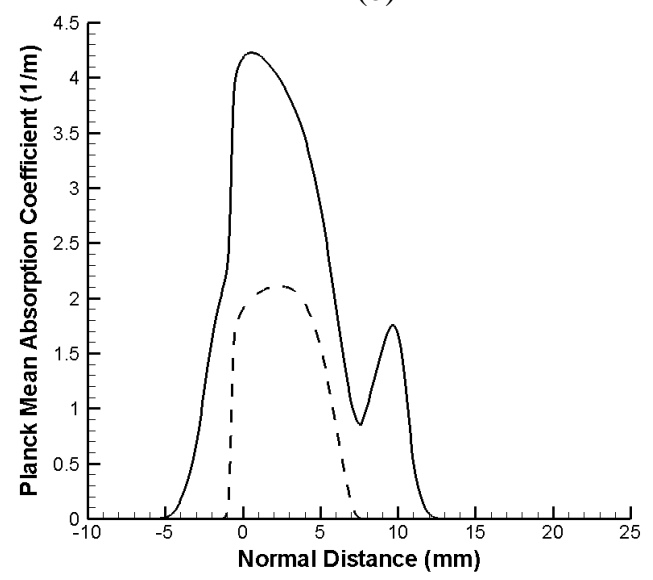

(d)

Fig. 2. Typical structure of an ethylene-air SLF flame. Spatial variations of: (a) mixture fraction; (b) temperature; (c) soot volume fraction; (d) Planck mean absorption coefficient. Fig. 2d compares the variations of the total mean absorption coefficient (with contributions due to $\mathrm{CO}_{2}, \mathrm{H}_{2} \mathrm{O}$, and soot, solid line) to those of its soot component (dashed line). Case, $\alpha=20 \mathrm{~s}^{-1}\left(\chi_{\mathrm{st}}=0.62 \mathrm{~s}^{-1}\right)$. 
fuel side, $x<-5 \mathrm{~mm}$; mixture fraction is equal to 0 on the oxidizer side, $x>10 \mathrm{~mm}$; and the flame is the region where mixture fraction takes values between 0 to 1 . The flame presented in Fig. 2 corresponds to a moderate value of strain rate $\alpha, \alpha=20 \mathrm{~s}^{-1}$ (by convention, $\alpha$ is measured on the oxidizer side of the flame), or equivalently to a moderate value of flame stretch $\chi_{s t}, \chi_{s t}=0.62 \mathrm{~s}^{-1}$. The thickness of the flame is approximately $15 \mathrm{~mm}$.

Figure $2 \mathrm{~b}$ presents the corresponding spatial variations of temperature. The peak value of flame temperature is $1965 \mathrm{~K}$. Note that the flame is significantly affected by radiant losses: in the absence of thermal radiation, the peak value of flame temperature is $2340 \mathrm{~K}$, which shows that in Fig. 2b, radiation is responsible for a $375 \mathrm{~K}$ decrease in peak temperature (the radiant fraction, defined as the spatially-averaged radiative cooling rate divided by the spatially-averaged combustion heat release rate, is $28 \%$ ).

Figure 2c presents the spatial variations of soot volume fraction. The peak value of soot volume fraction is approximately $1.1 \mathrm{ppm}$. In a SLF flame, the soot particles are formed and oxidized on the fuel side of the flame; also the particles are transported by convection and thermophoresis, and cannot cross to the oxidizer side of the flame (in Fig. 2c, there is no soot in the region $x>7.5 \mathrm{~mm}$ ). Soot particles impact the flame structure via their contribution on thermal radiation properties, and in particular the Planck mean absorption coefficient. The total mean absorption coefficient is the sum of a gas component due to $\mathrm{CO}_{2}$ and $\mathrm{H}_{2} \mathrm{O}$, and a soot particles component. Figure 2d compares the variations of the total mean absorption coefficient to those of its soot component. The figure shows that the gas and soot contributions are comparable in magnitude. The area under the solid curve in Fig. $2 \mathrm{~d}$ is the flame optical thickness; the optical thickness is approximately $3.5 \%$, which indicates that the flame is optically thin.

\section{FLAME RESPONSE TO CHANGES IN FLAME STRETCH}

We now turn to the response of SLF flames to changes in flame stretch. Figure 3 presents the variations of flame temperature with strain rate $\alpha$. The figure shows that the flammable domain of SLF flames is limited by upper and lower limits at high and low values of $\alpha$. The upper limit corresponds to the classical flame response to increasing mixing rates, i.e. to an intensification of combustion at moderate-to-high values of $\alpha$, followed by aerodynamic quenching once $\alpha \geq \alpha^{U L}$ ( $\chi_{s t} \geq \chi_{s t}^{U L}$ ). This upper limit is the only flame extinction limit observed under adiabatic combustion conditions (i.e., without radiation). The lower limit corresponds to the flame response to decreasing mixing rates, i.e., to a progressive weakening of combustion at moderate-to-low values of $\alpha$, followed by radiation-driven thermal quenching once $\alpha \leq \alpha^{L L}$ $\left(\chi_{s t} \leq \chi_{\text {st }}^{L L}\right)$. We find: $\alpha^{L L} \approx 0.8 \mathrm{~s}^{-1}\left(\chi_{s t}^{L L} \approx 0.025 \mathrm{~s}^{-1}\right)$ and $\alpha^{U L} \approx 1955 \mathrm{~s}^{-1}\left(\chi_{s t}^{U L} \approx 61 \mathrm{~s}^{-1}\right)$. Note that the extinction values of flame temperature are quite different at the two limits: $T_{s t}^{L L} \approx 1115 \mathrm{~K}$ and $T_{s t}^{U L} \approx 1720 \mathrm{~K}$; these results indicate that the flame extinction criterion cannot be cast as a critical flame temperature condition.

Figure 4 adopts a slightly different perspective in which the Damköhler number $D a$ is used as a convenient measure of flame strength. In the AEA analysis, $D a$ is defined as [14]:

$D a=\frac{8 \rho_{\infty}{ }^{(p+q-1)}\left(M_{s t} / M_{\infty}\right)^{(p+q-1)} T_{\infty}{ }^{(p+q-1)} r_{s}^{q+2} A}{\left(10^{6}\right)^{p+q-1} M_{F}{ }^{(p-1)} M_{O_{2}}{ }^{q} T_{a}{ }^{(p+q+1)}\left(\Delta H_{F} / c_{p}\right)^{(p+q+1)}\left(r_{s}+Y_{O_{2}, \infty}\right)^{2}} \times \frac{T_{s t}{ }^{(p+q+3)} \exp \left(-T_{a} / T_{s t}\right)}{\chi_{s t}}$

where $\rho_{\infty}, M_{\infty}$, and $T_{\infty}$ designate the mass density, molecular weight, and temperature of air at ambient conditions, $M_{s t}$ the molecular weight of the reactive mixture at flame conditions, $r_{s}$ the stoichiometric oxygen-to-fuel mass ratio, $A, p, q$, and $T_{a}$ model coefficients associated with the Arrhenius-like single-step finite-rate ethylene-air global chemistry model ( $A$ is the pre-exponential factor, $p$ and $q$ the fuel and oxygen concentration exponents, $T_{a}$ the activation temperature) [14,15], $M_{F}$ and $M_{O 2}$ the molecular weight of fuel (ethylene) and oxygen, $\Delta H_{F}$ the heat of combustion (per unit mass of fuel), $c_{p}$ the heat capacity (assumed constant), and $Y_{O 2, \infty}$ the mass fraction of oxygen in air. 


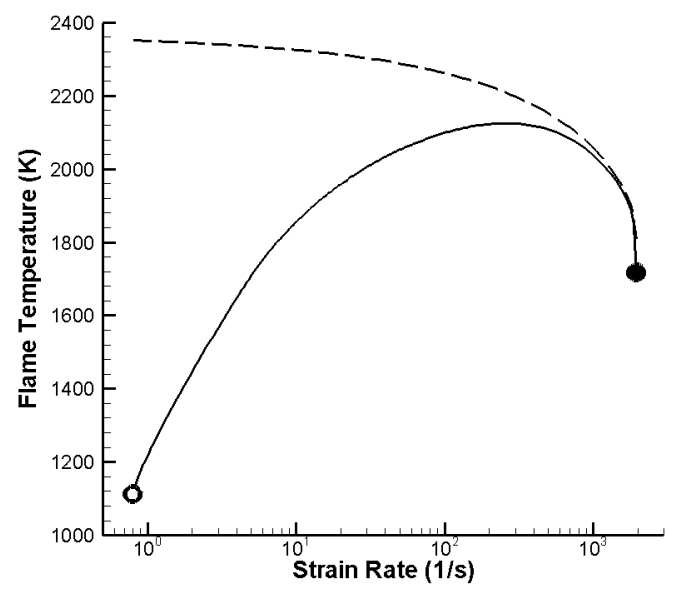

Fig. 3. Flame temperature $T_{\text {st }}$ versus strain rate $\alpha$. Solid line: SLF flame; dashed line: SLF flame without radiation (adiabatic flame). The circles at the end points of the curves mark the extinction limits. Two limits are observed in the presence of radiant losses: a fast mixing limit (solid circle on the right) and a slow mixing limit (open circle on the left); the slow mixing limit is not observed in the case of adiabatic combustion.

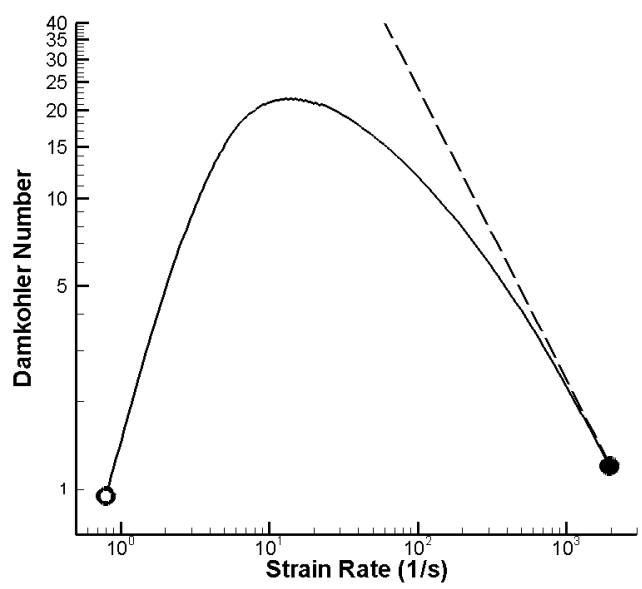

Fig. 4. Damköhler number $D a$ versus strain rate $\alpha$ (log-log plot). Solid line: SLF flame; dashed line: SLF flame without radiation (adiabatic flame). The circles at the end points of the curves mark the extinction limits; the critical values of $D a$ at the extinction limits are close to 1.

The RHS of Eq. 1 is written as the product of two factors: the first factor is essentially a constant; the second factor is a function of flame temperature, $T_{s t}$, and flame stretch, $\chi_{s t}$. We can now refine the scaling argument discussed previously and write:

$D a=C \times \frac{T_{s t}^{(p+q+3)} \exp \left(-T_{a} / T_{s t}\right)}{\chi_{s t}}$

where $C$ is a known constant, $p=0.1, q=1.65$, and $T_{a}=15107 \mathrm{~K}$ [15].

Figure 4 presents the variations of the Damköhler number $D a$ with strain rate $\alpha$. At intermediate values of $\alpha, \alpha \approx 10-100 \mathrm{~s}^{-1}, D a$ takes large values and the flame is strong, while at low or high values of $\alpha, D a$ takes low values and the flame is weak. Consistent with classical laminar flame theory, Fig. 4 shows that flame extinction occurs when $D a$ takes critically low values. An important result in Fig. 4 is that the critical 
values of $D a$ are of order 1 and are approximately equal at both lower and upper limits: $D a_{c}^{L L} \approx 0.95$ and $D a_{c}^{U L} \approx 1.2$.

\section{FLAME RESPONSE TO CHANGES IN FUEL AND OXIDIZER STREAMS COMPOSITION}

Figures 3 and 4 show that SLF flames are susceptible to aerodynamic quenching (for large flame stretch values) and radiation-driven thermal quenching (for low flame stretch values). Next, we turn to the topic of dilution quenching. We consider SLF flames with arbitrary levels of nitrogen added to the fuel or oxidizer side of the flame. We note $Y_{F, 1}\left(Y_{O 2,2}\right)$ the fuel (oxygen) mass fraction in the fuel (oxidizer) supply stream; we also note $T_{1}\left(T_{2}\right)$ the temperature of the fuel (oxidizer) supply stream. In a first step, $T_{1}$ and $T_{2}$ remain at ambient conditions, $T_{1}=T_{2}=300 \mathrm{~K}$.

Figure 5 presents the variations of the Damköhler number $D a$ with strain rate $\alpha$ for different levels of air dilution. The AEA analysis indicates that increased levels of air dilution result in a significant decrease of the size of the flammable domain: $\alpha^{L L} \approx 0.8 \mathrm{~s}^{-1}$ and $\alpha^{U L} \approx 1955 \mathrm{~s}^{-1}\left(Y_{O 2,2}=23.3 \%\right), \alpha^{L L} \approx 1 \mathrm{~s}^{-1}$ and $\alpha^{U L} \approx 570 \mathrm{~s}^{-1}\left(Y_{O 2,2}=20 \%\right), \alpha^{L L} \approx 1.5 \mathrm{~s}^{-1}$ and $\alpha^{U L} \approx 130 \mathrm{~s}^{-1}\left(Y_{O 2,2}=17 \%\right)$. These results point to the existence of a critical value of oxygen level in the oxidizer stream below which combustion cannot be sustained, regardless of the value of flame stretch; this critical value of $Y_{O 2,2}$ is further discussed in the next section.

Figure 6 presents the variations of the Damköhler number $D a$ with strain rate $\alpha$ for different levels of fuel dilution. The AEA analysis indicates that increased levels of fuel dilution result in a significant decrease of the size of the flammable domain: $\alpha^{L L} \approx 0.8 \mathrm{~s}^{-1}$ and $\alpha^{U L} \approx 1955 \mathrm{~s}^{-1}\left(Y_{F, 1}=100 \%\right), \alpha^{L L} \approx 1.3 \mathrm{~s}^{-1}$ and $\alpha^{U L} \approx 1075 \mathrm{~s}^{-1}\left(Y_{F, 1}=30 \%\right), \alpha^{L L} \approx 3.1 \mathrm{~s}^{-1}$ and $\alpha^{U L} \approx 80 \mathrm{~s}^{-1}\left(Y_{F, 1}=10 \%\right)$. These results point to the existence of a critical value of fuel level in the fuel stream below which combustion cannot be sustained, regardless of the value of flame stretch; this critical value of $Y_{F, 1}$ is further discussed in the next section.

The results presented in Figs. 5 and 6 are qualitatively similar. A careful comparison reveals that the SLF flames are more sensitive to air dilution than fuel dilution: if one accepts that the cases $\left(Y_{O 2,2}=17 \%\right.$, $\left.Y_{F, 1}=100 \%\right)$ and $\left(Y_{O 2,2}=23.3 \%, Y_{F, 1}=10 \%\right)$ have flammable domains of comparable sizes in strain rate space, one may conclude that it takes a $90 \%$ decrease in $Y_{F, 1}$ to provide the same flame weakening results as those observed with a $30 \%$ decrease in $Y_{O 2,2}$. This higher sensitivity of diffusion flames to oxygen level rather than fuel level is a direct consequence of the combustion stoichiometry (i.e., a consequence of the non-unity value of the stoichiometric oxygen-to-fuel mass ratio $r_{s}$ ).

Another important result in Figs. 5 and 6 is that the critical values of $D a$ are of order 1 and are approximately equal at both lower and upper limits; we find that $0.8 \leq D a_{c}^{L L} \leq 0.95$ and $0.8 \leq D a_{c}^{U L} \leq 1.2$. This result will be exploited in the next section when constructing flammability maps.

\section{FLAMMABILITY MAPS}

\section{Flame Extinction Criterion}

The AEA analysis presented previously shows that flame extinction corresponds to a critical value of the Damköhler number. Extinction is observed when:

$D a=C \times \frac{T_{s t}^{(p+q+3)} \exp \left(-T_{a} / T_{s t}\right)}{\chi_{s t}} \leq D a_{c}$

where $D a_{c}$ is the critical value of $D a$ at the extinction limit. 


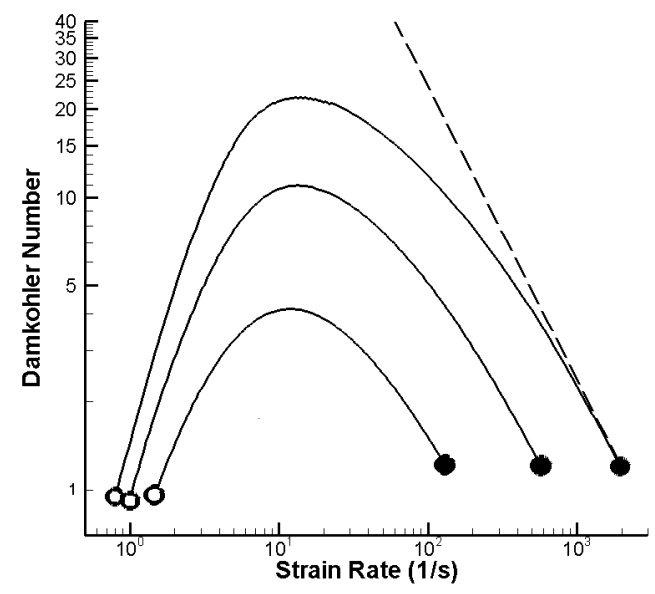

Fig. 5. Damköhler number $D a$ versus strain rate $\alpha$ (log-log plot) for different levels of air dilution. Top dashed line: SLF flame with $Y_{O 2,2}=23.3 \%$ (air conditions) and without radiation (adiabatic flame). Three solid lines: SLF flames with $Y_{O 2,2}=23.3 \%$ (top), $20 \%$ (middle), $17 \%$ (bottom). The circles at the end points of the curves mark the extinction limits; the critical values of $D a$ at the extinction limits are close to 1 .

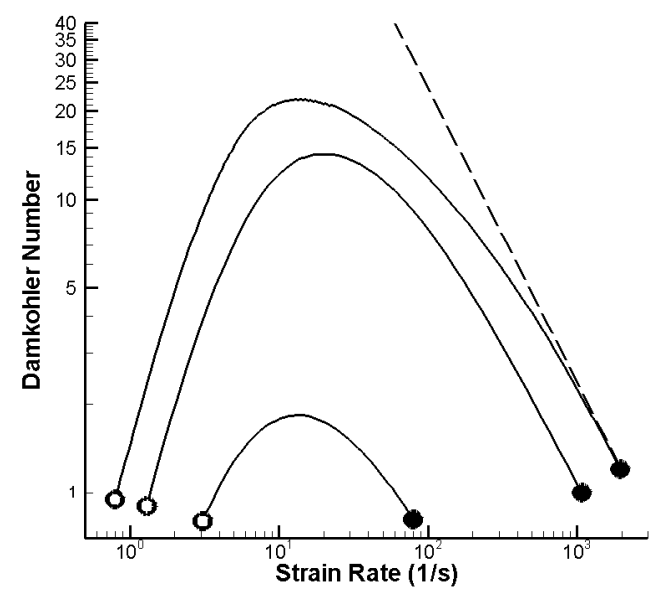

Fig. 6. Damköhler number $D a$ versus strain rate $\alpha$ (log-log plot) for different levels of fuel dilution. Top dashed line: SLF flame with $Y_{F, 1}=100 \%$ (air conditions) and without radiation (adiabatic flame). Three solid lines: SLF flames with $Y_{F, 1}=100 \%$ (top), $30 \%$ (middle), $10 \%$ (bottom). The circles at the end points of the curves mark the extinction limits; the critical values of $D a$ at the extinction limits are close to 1 .

As seen in Figs. 4-6 (see also Ref. [14]), the extinction limits correspond to quasi-unity values of $D a_{c}$, $0.8 \leq D a_{c} \leq 1.2$. This result lends support to the unifying concept of a flame Damköhler number as the basis to predict all flame extinction limits. In the following, we use an approximate expression for the flame extinction criterion: $D a_{c}=1$. Equation 3 suggests that extinction conditions can be simply parametrized by the flame stretch $\chi_{\text {st }}$ and the flame temperature $T_{s t}$. Figure 7 presents this two-dimensional flammability map with $\chi_{s t}$ and $T_{s t}$ as coordinates.

In Fig. 7, the flame stretch represents the effects of turbulent eddies, while the flame temperature contains information on the composition and heat content of the fuel and oxidizer supply streams as well as on the magnitude of heat losses. We now turn to a description of the flame temperature $T_{s t}$. In the AEA analysis, $T_{s t}$ is a leading-order flame temperature (i.e., a Burke-Schumann temperature modified by thermal radiation or other external heat losses) and may be expressed as [14]: 


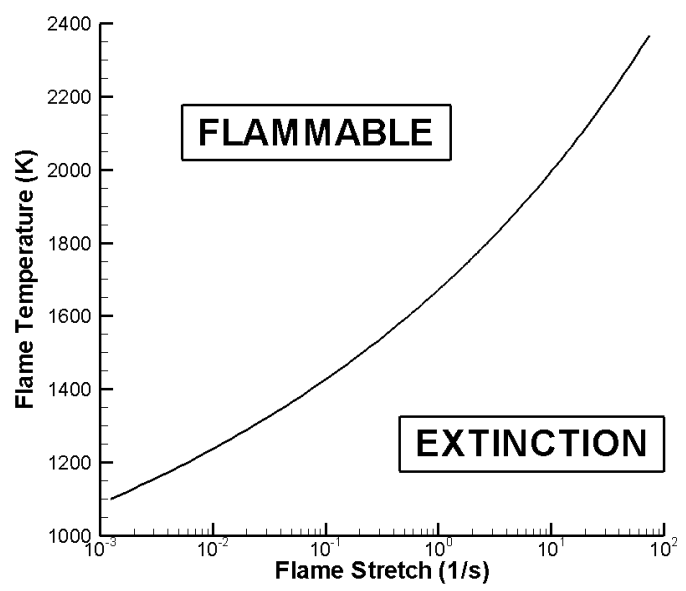

Fig. 7. Flammability map using flame stretch, $\chi_{s t}$, and flame temperature, $T_{s t}$, as coordinates. The solid line corresponds to the extinction limit as obtained from AEA theory, $D a=1$ in Eq. 3. The flammable domain corresponds to the upper-left region; the flame extinction domain corresponds to the lower-right region.

$T_{s t}=\frac{Y_{O_{2}, 2}}{r_{s} Y_{F, 1}+Y_{O_{2}, 2}} T_{1}+\frac{r_{s} Y_{F, 1}}{r_{s} Y_{F, 1}+Y_{O_{2}, 2}} T_{2}+\frac{Y_{O_{2}, 2} Y_{F, 1}}{r_{s} Y_{F, 1}+Y_{O_{2}, 2}}\left(\frac{\Delta H_{F}}{c_{p}}\right)\left(1+H_{s t}\right)$

where $H_{s t}$ is an excess enthalpy variable introduced to provide a measure of the magnitude of non-adiabatic effects (in the present problem, $H_{s t} \leq 0$ so that one should interpret $H_{s t}$ as an enthalpy deficit variable). We have:

$$
H_{s t}=\frac{\left(T_{s t}-T_{s t}^{a d}\right)}{\frac{Y_{O_{2}, 2} Y_{F, 1}}{r_{s} Y_{F, 1}+Y_{O_{2}, 2}}\left(\frac{\Delta H_{F}}{c_{p}}\right)}
$$

where $T_{s t}^{a d}$ is the classical Burke-Schumann flame temperature obtained under adiabatic combustion conditions, $T_{s t}^{a d}=\left[Y_{O_{2}, 2} T_{1}+\left(r_{s} Y_{F, 1}\right) T_{2}+\left(Y_{O_{2}, 2} Y_{F, 1}\right)\left(\Delta H_{F} / c_{p}\right)\right] /\left(r_{s} Y_{F, 1}+Y_{O_{2}, 2}\right)$. In Eq. 5, the denominator of the RHS corresponds to the contribution of heat release to flame temperature. With this definition, $H_{s t}$ is a non-dimensional variable that takes values between $(-1)$ and $0 ; H_{s t}=0$ under adiabatic burning conditions while $H_{s t}=-1$ under non-burning conditions.

The flame extinction criterion may now be re-written as:

$$
\begin{aligned}
& \frac{C}{\chi_{s t}} \times\left[\frac{\left.Y_{O_{2}, 2} T_{1}+\left(r_{s} Y_{F, 1}\right) T_{2}+\left(Y_{O_{2}, 2} Y_{F, 1}\right)\left(\Delta H_{F} / c_{p}\right)\left(1+H_{s t}\right)\right)}{\left(r_{s} Y_{F, 1}+Y_{O_{2}, 2}\right)}\right]^{(p+q+3)} \\
& \times \exp \left[-\frac{T_{a}\left(r_{s} Y_{F, 1}+Y_{O_{2}, 2}\right)}{Y_{O_{2}, 2} T_{1}+\left(r_{s} Y_{F, 1}\right) T_{2}+\left(Y_{O_{2}, 2} Y_{F, 1}\right)\left(\Delta H_{F} / c_{p}\right)\left(1+H_{s t}\right)}\right]=1
\end{aligned}
$$

Equation 6 shows that extinction conditions can be parametrized by the following six input variables: $\chi_{\text {st }}$, $H_{s t}, Y_{O 2,2}, T_{2}, Y_{F, 1}$, and $T_{1}$. The parameter $\chi_{s t}$ represents the effects of the flow field; $H_{s t}$ represents the effects of thermal losses (by radiant cooling, convective cooling to cold wall surfaces, water evaporative cooling in fire suppression applications, etc); $Y_{O 2,2}$ and $T_{2}$ represent air vitiation effects (i.e., dilution and preheating of the oxidizer supply stream); $Y_{F, 1}$ and $T_{1}$ represent fuel vitiation effects (i.e., dilution and 


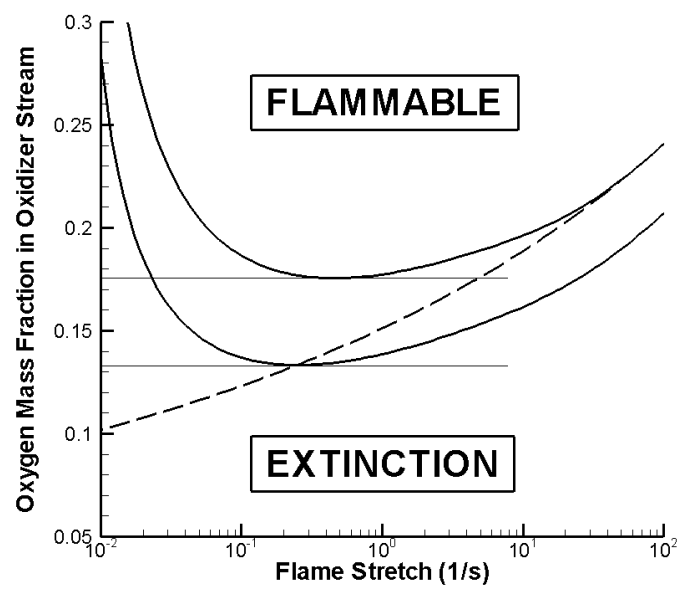

Fig. 8. Flammability map using flame stretch, $\chi_{s t}$, and oxygen level in the oxidizer supply stream, $Y_{O 2,2}$, as coordinates. The two (thick) solid lines and the dashed line correspond to three extinction limits. Top solid line: SLF flame $\left(H_{s t}=H_{s t}^{S L F}\left(\chi_{s t}\right)\right)$ with $T_{2}=300 \mathrm{~K}$; lower solid line: SLF flame $\left(H_{s t}=H_{s t}^{S L F}\left(\chi_{s t}\right)\right)$ with

$T_{2}=600 \mathrm{~K}$; lower dashed line: adiabatic flame $\left(H_{s t}=0\right)$ with $T_{2}=300 \mathrm{~K}$. The flammable domain corresponds to the upper region in the map; the flame extinction domain corresponds to the lower region.

The two (thin) horizontal lines mark the location of the oxygen-based lower flammability limit,

$$
Y_{O_{2}, 2}=Y_{O_{2}, 2}^{c} \text {, for } T_{2}=300 \mathrm{~K} \text { (top) and } 600 \mathrm{~K} \text { (bottom). }
$$

preheating of the fuel supply stream). Extinction is promoted by large negative values of $H_{s t}$ as well as by low values of $Y_{O 2,2}, T_{2}, Y_{F, 1}$, and $T_{1}$. The effect of the flame stretch $\chi_{s t}$ in Eq. 6 is non-trivial because in general, variations in flame stretch also induce variations in the heat loss parameter (in many problems, $\chi_{s t}$ and $H_{\text {st }}$ are not independent quantities).

In the following two sections, we use Eq. 6 and present two-dimensional flammability maps using different assumptions aimed at reducing the dimension of the parameter space from six to two.

\section{Flammability Map for SLF Flames with a Focus on Air Vitiation Effects}

Figure 8 presents a two-dimensional flammability map with $\chi_{s t}$ and $Y_{O 2,2}$ as coordinates, and with $T_{2}$ as a parameter $\left(T_{2}=300 \mathrm{~K}\right.$ or $\left.600 \mathrm{~K}\right)$. The fuel stream properties $Y_{F, 1}$ and $T_{1}$ are fixed, $Y_{F, 1}=1$ and $T_{1}=300 \mathrm{~K}$; the excess enthalpy variable $H_{s t}$ is either 0 (adiabatic case) or is described as a prescribed function of flame stretch $H_{s t}^{S L F}\left(\chi_{s t}\right)$. The function $H_{s t}^{S L F}\left(\chi_{s t}\right)$ is obtained using the AEA results previously discussed; $H_{s t}^{S L F}\left(\chi_{s t}\right)$ represents the radiant losses experienced by a SLF flame. Note that by using $H_{s t}^{S L F}\left(\chi_{s t}\right)$, we assume that air-diluted flames have losses that are similar to those observed in non-diluted flames.

Figure 8 shows that as expected, the flammable domain is larger for $T_{2}=600 \mathrm{~K}$ than that obtained for $T_{2}=300 \mathrm{~K}$. For both cases, the extinction limit has a characteristic U-shape in the $\left(\chi_{s t}, Y_{O 2,2}\right)$ plot: the flammable domain is large for large values of $Y_{O 2,2}$ and becomes increasingly small as $Y_{O 2,2}$ is decreased; for $Y_{O 2,2}$ below a certain critical value, noted $Y_{O_{2}, 2}^{c}$, the flammable domain vanishes and combustion cannot be sustained, regardless of the value of flame stretch (a result that was anticipated in the discussion of Figs. 5 and 6).

We find: $Y_{O_{2}, 2}^{c} \approx 17.5 \%$ for $T_{2}=300 \mathrm{~K}$ and $Y_{O_{2}, 2}^{c} \approx 13.5 \%$ for $T_{2}=600 \mathrm{~K}$. The value $Y_{O_{2}, 2}^{c}$ may be interpreted as an oxygen-based lower flammability limit for diffusion flames; it may also be viewed as a rigorous definition of a limiting oxygen index (LOI) for SLF flames. 


\section{Flammability Map for SLF Flames with a Focus on Fuel Vitiation Effects}

Figure 9 presents a two-dimensional flammability map with $\chi_{s t}$ and $Y_{F, 1}$ as coordinates, and with $T_{1}$ as a parameter $\left(T_{1}=300 \mathrm{~K}\right.$ or $\left.600 \mathrm{~K}\right)$. The oxidizer stream properties $Y_{O 2,2}$ and $T_{2}$ are fixed, $Y_{O 2,2}=23.3 \%$ and

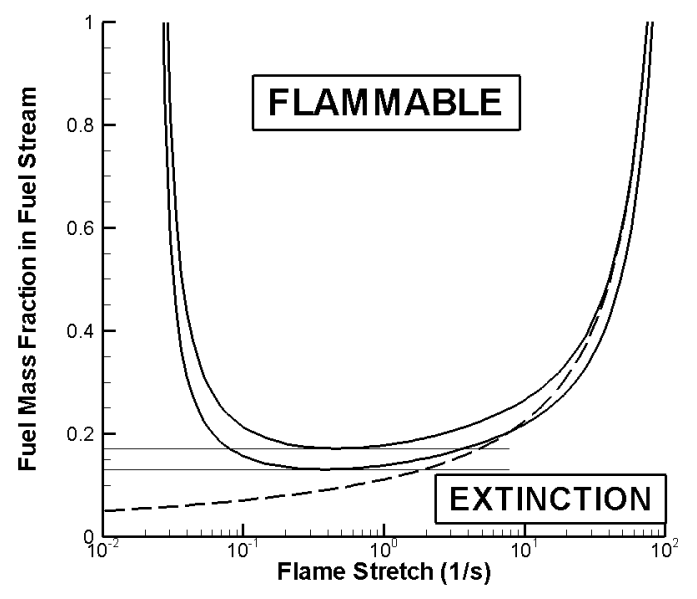

Fig. 9. Flammability map using flame stretch, $\chi_{\text {st }}$, and fuel level in the fuel supply stream, $Y_{F, 1}$, as coordinates. The two (thick) solid lines and the dashed line correspond to three extinction limits. Top solid line: SLF flame $\left(H_{s t}=H_{s t}^{S L F}\left(\chi_{s t}\right)\right)$ with $T_{1}=300 \mathrm{~K}$; lower solid line: SLF flame $\left(H_{s t}=H_{s t}^{S L F}\left(\chi_{s t}\right)\right)$ with

$T_{1}=600 \mathrm{~K}$; lower dashed line: adiabatic flame $\left(H_{s t}=0\right)$ with $T_{1}=300 \mathrm{~K}$. The flammable domain corresponds to the upper-central region in the map; the flame extinction domain corresponds to the lower and right/left regions. The two (thin) horizontal lines mark the location of the fuel-based lower flammability limit, $Y_{F, 1}=Y_{F, 1}^{c}$, for $T_{1}=300 \mathrm{~K}$ (top) and $600 \mathrm{~K}$ (bottom).

$T_{2}=300 \mathrm{~K}$; the excess enthalpy variable $H_{s t}$ is either 0 (adiabatic case) or is described as a prescribed function of flame stretch $H_{s t}^{S L F}\left(\chi_{s t}\right)$. The function $H_{s t}^{S L F}\left(\chi_{s t}\right)$ is obtained using the AEA results previously discussed. Note that by using $H_{s t}^{S L F}\left(\chi_{s t}\right)$, we assume that fuel-diluted flames have losses that are similar to those observed in non-diluted flames.

Figure 9 shows that as expected, the flammable domain is larger for $T_{1}=600 \mathrm{~K}$ than that obtained for $T_{1}=300 \mathrm{~K}$. For both cases, the extinction limit has a characteristic U-shape in the $\left(\chi_{s t}, Y_{F, 1}\right)$ plot: the flammable domain is large for large values of $Y_{F, 1}$ and becomes increasingly small as $Y_{F, 1}$ is decreased; for $Y_{F, 1}$ below a certain critical value, noted $Y_{F, 1}^{c}$, the flammable domain vanishes and combustion cannot be sustained, regardless of the value of flame stretch (a result that was anticipated in the discussion of Figs. 5 and 6). We find: $Y_{F, 1}^{c} \approx 17 \%$ for $T_{1}=300 \mathrm{~K}$ and $Y_{F, 1}^{c} \approx 13 \%$ for $T_{1}=600 \mathrm{~K}$. The value $Y_{F, 1}^{c}$ may be interpreted as a fuel-based lower flammability limit for diffusion flames.

\section{Treatment of Flame Extinction in CFD Models}

We now turn to a brief discussion of the application of the flame extinction criterion proposed in Eq. 6 to computational fluid dynamics (CFD) based fire models. Figs. 7-9 illustrate some of the current challenges found in combustion modeling since it is shown that flame extinction is controlled by flame-based quantities, e.g., stretch and temperature, that are unresolved in a CFD calculation. A direct application of the flame extinction model in Eq. 6 to CFD is possible provided that the six input variables $\chi_{s t}, H_{s t}, Y_{O 2,2}$, $T_{2}, Y_{F, 1}$, and $T_{1}$, are described with suitable spatial and temporal resolution. This question is beyond the scope of the present paper and will be the focus of future work.

In current CFD fire models, the treatment of flame extinction is much simplified and is usually based on a rough version of the flammability map presented in Fig. 7. For instance, we consider in the following the fire model called Fire Dynamics Simulator (FDS); FDS is developed by the Building and Fire Research 
Laboratory of the US National Institute of Standards and Technology, see Ref. [16] for details. In FDS, flame extinction is assumed to be dominated by air vitiation effects (associated with variations in $Y_{O 2,2}$ and $T_{2}$ ). Extinction by aerodynamic quenching (associated with variations in $\chi_{\mathrm{st}}$ ) and by fuel vitiation effects (associated with variations in $Y_{F, 1}$ and $T_{1}$ ) are neglected; extinction by thermal quenching (associated with

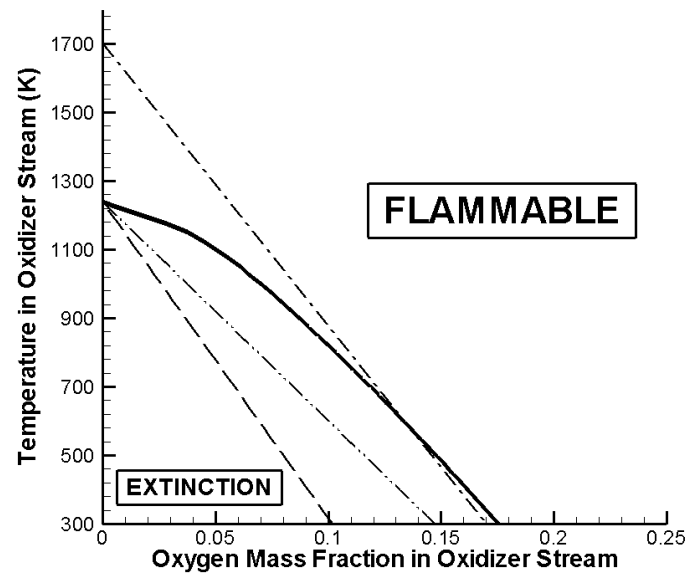

Fig. 10. Flammability map using properties of the oxidizer supply stream, oxygen level $Y_{O 2,2}$ and temperature $T_{2}$, as coordinates. The lines correspond to four different estimates of the extinction limit. Top dash-dot line: FDS model; middle (thick) solid line: AEA model (Eq. 6) with $H_{s t}=H_{s t}^{S L F}\left(\chi_{s t}\right)$; middle dash-dot-dot line: AEA model (Eq. 6) with $H_{s t}=-0.3$; bottom dashed line: AEA model (Eq. 6) with $H_{s t}=0$

(adiabatic flame). The flammable domain corresponds to the upper-right region in the map; the flame extinction domain corresponds to the lower-left region.

variations in $H_{s t}$ ) is implicitly accounted for via calibration factors but the treatment is questionable since $H_{s t}$ is not treated as a problem-dependent variable $[17,18]$.

Figure 10 presents a two-dimensional flammability map with $\chi_{s t}$ and $Y_{O 2,2}$ as coordinates. In Fig. 10, the FDS flame extinction model is compared to four different versions of the AEA model given by Eq. 6 . Note that we consider here the original version of the FDS model, as described in Refs. [17,18], which assumes constant heat capacities; see Ref. [19] for a recently modified version of the FDS flame extinction model that accounts for variable heat capacities and is formulated as an enthalpy-based criterion. In order to allow direct comparisons between FDS and AEA, and since the FDS model does not account for effects of flame stretch, the AEA model uses the oxygen-based lower flammability limit $Y_{O_{2}, 2}^{c}$ as controlling variable and construct the extinction line from a series of calculations in which $Y_{O_{2}, 2}^{c}$ is determined for different values of $T_{2}$ (for instance, in Fig. 8, $Y_{O_{2}, 2}^{c}\left(T_{2}=300 \mathrm{~K}\right) \approx 17.5 \%$ and $Y_{O_{2}, 2}^{c}\left(T_{2}=600 \mathrm{~K}\right) \approx 13.5 \%$ ). This treatment has the advantage of bypassing flame stretch as one of the controlling variables in Eq. 6; the drawback is that it provides an overestimate of the size of the flammable domain (i.e., an under-estimate of the probability of occurrence of local flame extinction events).

Figure 10 shows that the FDS model compares well with the version of the AEA model in which heat losses are estimated from the radiant losses experienced by a SLF flame, $H_{s t}=H_{s t}^{S L F}\left(\chi_{s t}\right)$. However, in flame systems in which heat losses deviate significantly from the SLF flame model adopted in AEA, for instance in flame systems with high soot loading and significant multi-dimensional effects [14] and/or in systems with fire suppressants, the assumption $H_{s t}=H_{s t}^{S L F}\left(\chi_{s t}\right)$ is of questionable accuracy. For these systems, the heat loss parameter $H_{s t}$ cannot be prescribed and must be treated as a variable.

\section{CONCLUSION}

The effect of flame stretch, heat losses, and fuel and air vitiation on the extinction limits of ethylene-air diffusion flames are studied using large activation energy asymptotic (AEA) theory. The AEA analysis 
includes: a phenomenological soot model that accounts for particles inception, growth and oxidation, and a generalized treatment of thermal radiation that accounts for both emission and absorption phenomena and applies to participating media ranging from optically-thin to optically-thick [14].

The AEA analysis leads to a critical-Damköhler-number-based flame extinction criterion. The critical Damköhler number criterion is formulated in terms of six input variables; these variables characterize the magnitude of flame stretch, the magnitude of the flame heat losses, and the composition and heat content of the fuel and oxidizer supply streams. The dimension of the parameter space to predict flame extinction is therefore equal to six. While we recommend treating flame extinction in its full complexity as a sixdimensional problem, the present paper considers different assumptions aimed at reducing the dimension of the parameter space from six to two and that lead to two-dimensional flammability maps. These flammability maps provide valuable insights; it is found for instance that diffusion flames are more sensitive to air vitiation than fuel vitiation.

A comparison is also made between the present AEA flame extinction model and the model currently available in the CFD fire model FDS. In FDS, flame extinction is assumed to be dominated by air vitiation effects; effects of flame stretch and fuel vitiation are neglected; and effects of heat losses are simplified. Consequently, the FDS model is two-dimensional, while the AEA model is six-dimensional. Results show that the FDS model compares well with one version of a two-dimensional AEA model in which heat losses are estimated from the radiant losses experienced by a laminar counter-flow flame. The comparison suggests that the FDS model provides an overestimate of the size of the flammable domain.

\section{ACKNOWLEDGMENTS}

This work was supported in part by the US National Science Foundation, Division of Chemical, Bioengineering, Environmental, and Transport Systems (Grant No. CTS-0553508) and Office of Cyberinfrastructure (PetaApps Program, Grant No. OCI-0904660).

\section{REFERENCES}

[1] Williams, F.A., Combustion Theory, 2nd Ed., Addison Wesley, 1985.

[2] Peters, N., Turbulent Combustion, Cambridge University Press, 2000.

[3] Law, C.K., Combustion Physics, Cambridge University Press, 2006.

[4] Chao, B.H., Law, C.K., T'ien, J.S., "Structure and extinction of diffusion flames with flame radiation,” Twenty-Third Symposium (International) on Combustion, Symposium (International) on Combustion, 1991, pp. 523-531, http://dx.doi.org/10.1016/S0082-0784(06)80299-7

[5] Daguse, T., Croonenbroek, T., Rolon, J.C., Darabiha, N., and Soufiani, A., (1996) Study of radiative effects on laminar counterflow $\mathrm{H}_{2} / \mathrm{O}_{2} / \mathrm{N}_{2}$ diffusion flames, Combustion and Flame 106: 271-287, http://dx.doi.org/10.1016/0010-2180(95)00251-0

[6] T'ien, J.S., Bedir, H., "Radiative extinction of diffusion flames - A review," Proceedings of the First Asia-Pacific Conference on Combustion, Osaka, Japan, 1997, pp. 345-352.

[7] Rhatigan, J.L., Bedir, H., T’ien, J.S., (1998) Gas-phase radiative effects on the burning and extinction of a solid fuel, Combustion and Flame 112: 231-241, http://dx.doi.org/10.1016/S00102180(97)81771-3

[8] Chan, S.H., Yin, J.Q., Shi, B.J., (1998) Structure and extinction of methane-air flamelet with radiation and detailed chemical kinetic mechanism, Combustion and Flame 112: 445-456, http://dx.doi.org/10.1016/S0010-2180(97)00133-8

[9] Maruta, K., Yoshida, M., Guo, H., Ju, Y., Niioka, T., (1998) Extinction of low-stretched diffusion flame in microgravity, Combustion and Flame 112: 181-187, http://dx.doi.org/10.1016/S00102180(97)81766-X

[10] Bai, X.S., Fuchs, L., Mauss, F., (2000) Laminar flamelet structure at low and vanishing scalar dissipation rate, Combustion and Flame 120: 285-300, http://dx.doi.org/10.1016/S00102180(99)00093-0 
[11] Liu, F., Smallwood, G.J., Gülder, Ö.L., Ju, Y., (2000) Asymptotic analysis of radiative extinction in counterflow diffusion flames of nonunity Lewis numbers, Combustion and Flame 121: 275287, http://dx.doi.org/10.1016/S0010-2180(99)00143-1

[12] Wang, H.Y., Chen, W.H., Law, C.K., (2007) Extinction of counterflow diffusion flames with radiative heat loss and nonunity Lewis numbers, Combustion and Flame 148: 100-116, http://dx.doi.org/10.1016/j.combustflame.2006.10.005

[13] Narayanan, P., Trouvé, A., (2009) Radiation-driven flame weakening effects in sooting turbulent diffusion flames, Proceedings of the Combustion Institute 32: 1481-1489, http://dx.doi.org/10.1016/j.proci.2008.06.056

[14] Narayanan, P., Baum, H.R., Trouvé, A., (2011) Effect of soot addition on extinction limits of luminous laminar counterflow diffusion flames, Proceedings of the Combustion Institute 33: 2539-2546, http://dx.doi.org/10.1016/j.proci.2010.07.003

[15] Westbrook, C.K., Dryer, F.L, (1981) Simplified reaction mechanisms for the oxidation of hydrocarbon fuels in flames, Combustion Science Technology 27: 31-43, http://dx.doi.org/10.1080/00102208108946970

[16] National Institute of Standards and Technology, Fire Dynamics Simulator, http://www.fire.nist.gov/fds/

[17] McGrattan, K., Baum, H., Rehm, R., Mell, W., McDermott, R., Hostikka, S., Floyd, J., "Fire Dynamics Simulator (Version 5). Technical Reference Guide, Volume 1: Mathematical Model,” National Institute of Standards and Technology Special Publication 1018-5, Gaithersburg, MD, 2010.

[18] Hu, Z., Utiskul, Y., Quintiere, J.G., Trouvé, A., (2007) Towards large eddy simulations of flame extinction and carbon monoxide emission in compartment fires, Proceedings of the Combustion Institute 31: 2537-2545, http://dx.doi.org/10.1016/j.proci.2006.08.053

[19] Vaari, J., Floyd, J., McDermott, R., (2011) CFD simulations on extinction of co-flow diffusion flames, Fire Safety Science 10: accepted for presentation. 